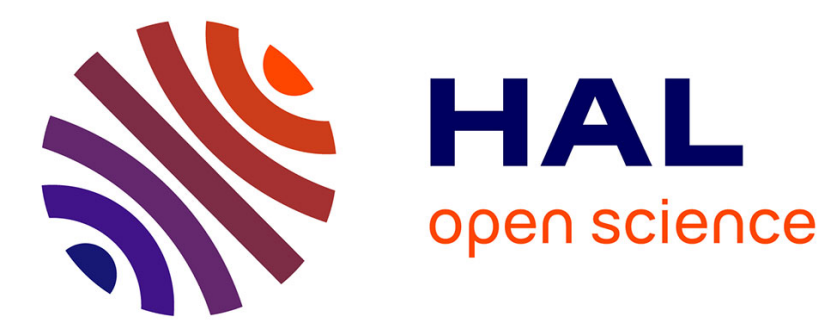

\title{
A micropillar for cavity optomechanics
}

A. G. Kuhn, M. Bahriz, O. Ducloux, C. Chartier, O. Le Traon, T. Briant, P. -F. Cohadon, A. Heidmann, Christine Michel, L. Pinard, et al.

\section{To cite this version:}

A. G. Kuhn, M. Bahriz, O. Ducloux, C. Chartier, O. Le Traon, et al.. A micropillar for cavity optomechanics. Applied Physics Letters, 2011, 99, pp.121103. 10.1063/1.3641871 . hal-00609698

\section{HAL Id: hal-00609698 \\ https://hal.science/hal-00609698}

Submitted on 19 Jul 2011

HAL is a multi-disciplinary open access archive for the deposit and dissemination of scientific research documents, whether they are published or not. The documents may come from teaching and research institutions in France or abroad, or from public or private research centers.
L'archive ouverte pluridisciplinaire HAL, est destinée au dépôt et à la diffusion de documents scientifiques de niveau recherche, publiés ou non, émanant des établissements d'enseignement et de recherche français ou étrangers, des laboratoires publics ou privés. 


\title{
A micropillar for cavity optomechanics
}

\author{
A. G. Kuhn, ${ }^{2, a)}$ M. Bahriz, ${ }^{1}$ O. Ducloux, ${ }^{1}$ C. Chartier, ${ }^{1}$ O. Le Traon, ${ }^{1, b)}$ T. Briant, ${ }^{2}$ P.-F. Cohadon, ${ }^{2}$ A. \\ Heidmann, ${ }^{2}$ C. Michel, ${ }^{3}$ L. Pinard, ${ }^{3}$ and R. Flaminio ${ }^{3}$ \\ 1) ONERA, Physics Department; 29 avenue de la Division Leclerc, 92322 Châtillon, \\ France \\ ${ }^{2)}$ Laboratoire Kastler Brossel, ENS, UPMC, CNRS; case 74, 4 place Jussieu, 75005 Paris, \\ France \\ 3) Laboratoire des Matériaux Avancés, CNRS, IN2P3; Bâtiment Virgo, 7 avenue Pierre de Coubertin, \\ 69622 Villeurbanne, France
}

\begin{abstract}
We present a new micromechanical resonator designed for cavity optomechanics. We have used a micropillar geometry to obtain a high-frequency mechanical resonance with a low effective mass and a very high quality factor. We have coated a $60-\mu \mathrm{m}$ diameter low-loss dielectric mirror on top of the pillar and are planning to use this micromirror as part of a high-finesse Fabry-Perot cavity, to laser cool the resonator down to its quantum ground state and to monitor its quantum position fluctuations by quantum-limited optical interferometry.
\end{abstract}

Reaching the quantum ground state of a macroscopic mechanical object is a major experimental challenge in physics, at the origin of the rapid emergence of the cavity optomechanics research field. Many groups have been targeting this objective for a decade ${ }^{1-4}$, using a wide range of resonators oscillating at frequencies from a few $\mathrm{Hz}$ to the $\mathrm{GHz}$-band ${ }^{1,3}$, and different techniques of displacement sensing $2,3,5,6$. The development of a very sensitive position sensor combined with a mechanical resonator in its ground state would have important consequences $^{7}$, not only for fundamental aspects in quantum physics such as entanglement ${ }^{8}$ and decoherence of mechanical resonators, but also for potential applications such as the detection of very weak forces.

Two conditions have to be fulfilled in order to reach and demonstrate the mechanical ground state. The thermal energy has to be small with respect to the zero-point quantum energy: $k_{\mathrm{B}} T_{\mathrm{c}} \ll h \nu_{\mathrm{m}}$. For a resonator oscillating at a frequency $\nu_{\mathrm{m}}=4 \mathrm{MHz}$, the resulting temperature $T_{\mathrm{c}}$ is in the sub-mK range and conventional cryogenic cooling has therefore to be combined with novel cooling mechanisms such as cavity cooling in a FabryPerot cavity ${ }^{4,9-11}$.

The second requirement is obviously to be able to detect the very small residual displacement fluctuations associated with the quantum ground state. The measurement sensitivity must be better than the expected displacement noise at resonance, which scales as

$\mathrm{S}_{x}\left[\nu_{\mathrm{m}}\right] \simeq\left(\frac{25 \mu \mathrm{g}}{M}\right)\left(\frac{Q_{\mathrm{c}}}{2 \times 10^{3}}\right)\left(\frac{4 \mathrm{MHz}}{\nu_{\mathrm{m}}}\right)^{2} 10^{-38} \mathrm{~m}^{2} / \mathrm{Hz}$,

where $M$ is the effective mass of the resonator and $Q_{\mathrm{c}}$ its mechanical quality factor. As all optical cooling mechanisms increase the damping, $Q_{\mathrm{c}}$ is here the final quality factor related to the intrinsic quality factor $Q$ of the resonator by $Q_{\mathrm{c}} T_{\mathrm{c}}=Q T$, where $T_{\mathrm{c}} / T$ is the cooling ratio.

\footnotetext{
a) Electronic mail: aurelien.kuhn@spectro.jussieu.fr

b) Electronic mail: Olivier.Le_Traon@onera.fr
}

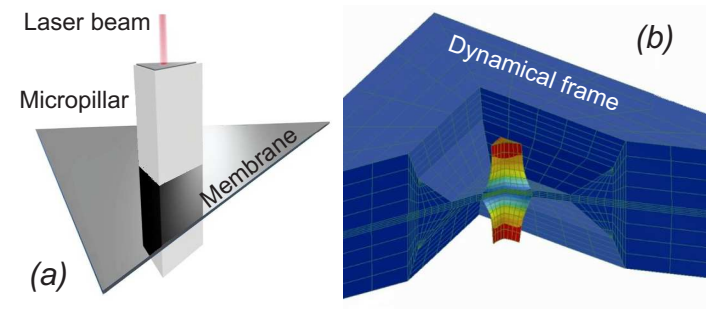

Figure 1. 3D artist view of the micropillar $(a)$ and finiteelement simulation of the displacement field of the first compression-expansion mode of the pillar $(b)$.

One thus may use a resonator with an ultra-high $Q$, larger than $10^{6}$, at a cryogenic temperature of $100 \mathrm{mK}$, in order to ensure a sufficient $Q_{\mathrm{c}} \simeq 1000$ at the final effective temperature.

In this letter, we present the mechanical design and experimental characterization of such a resonator. It consists in a micropillar mechanically decoupled from the wafer by a dynamical frame and clamped at its center by a thin membrane. A high-reflectivity mirror is coated on top of the pillar, thus providing a way for interferometric sensing of its motion and for future applications in cavity optomechanics. Advantages of such a pillar geometry are twofold. First, a compression-expansion mechanical mode of the pillar has a longitudinal node at its center, decreasing mechanical loss through the membrane. Second, the top area of the pillar has a quasi-null strain, reducing the influence of the relatively poor mechanical quality of the optical layers ${ }^{13}$ on the overall mechanical Q.

We present in the following the full design of the resonator using finite-element modelling (FEM) simulations, its microfabrication and experimental mechanical characterization (resonance frequency and $Q$ factor) using a Michelson-interferometer test bench.

As shown in Fig. 1, the basic structure is a $L=1 \mathrm{~mm}$ long and $240-\mu \mathrm{m}$ wide pillar made of crystalline quartz, clamped at its center to the external frame by a $20-\mu \mathrm{m}$ 

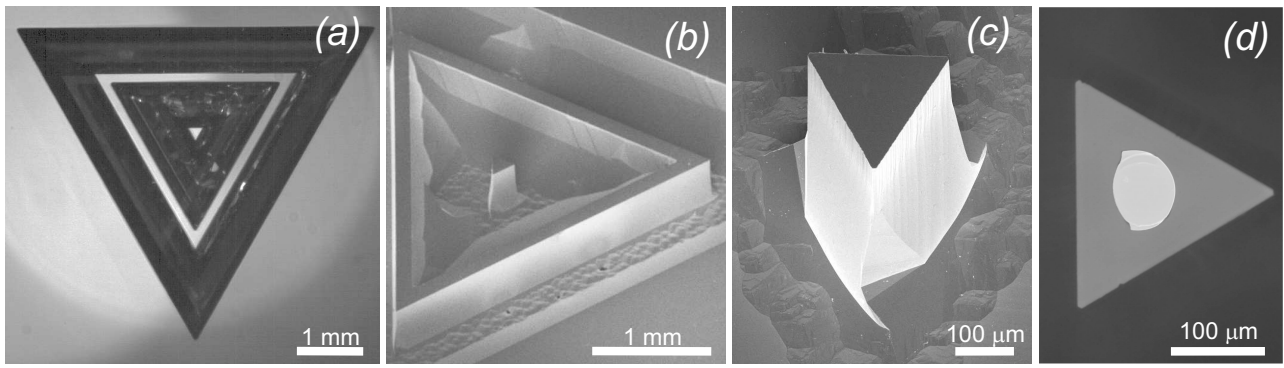

Figure 2. Optical view $(a)$ and scanning electron microscope picture $(b)$ of the full structure with the micropillar at the center, its clamping membrane and dynamical frame; closer view of the micropillar before the optical coating $(c)$, and top view of the coating $(d)$.

thin membrane. According to FEM simulations, with a bulk Young modulus $E=102.7 \mathrm{GPa}$ and a density $\rho=$ $2648 \mathrm{~kg} / \mathrm{m}^{3}$, resonance frequency and effective mass are expected around $3.2 \mathrm{MHz}$ and $25 \mu \mathrm{g}$ for the fundamental vibration mode.

We have chosen to use a single quartz crystal as bulk material to benefit from its high intrinsic quality factor, with a value $Q_{\text {int }} \simeq 5 \times 10^{6}$ expected at room temperature ${ }^{14,15}$, which can be increased by one to two orders of magnitude at cryogenic temperature ${ }^{16}$. Viscous air damping might be another limiting factor, but is experimentally negligible for pressures below $10^{-1} \mathrm{mBar}$.

As the effect of the mirror coated on top of the pillar is expected to be negligible as well, the main limitation to the quality factor is due to Poisson effects induced inside the membrane by the radial deformations of the pillar at the vibration node. A crystallographic orientation of the pillar along the $Z$ axis has been chosen, as well as a pillar equilateral cross-section with respect to the quartz trigonal symmetry: a nominally symmetric structure with regard to its median plane is thus possible, which is a sine qua non condition to obtain a good balancing of the length extension mode. In order to reduce mechanical losses, we have carefully designed an external dynamical frame $^{12}$ clamped to the wafer by another membrane (see Figs. $1 b, 2 a$ and $2 b$ ). The oscillation of the frame with an opposite phase then makes up for Poisson effects. The whole geometry has been optimized by FEM simulation (using SAMCEF code) to get an exact matching between the pillar and frame momenta, thus strongly decreasing the energy density at the location of the external membrane, and reducing clamping losses to a minimum. Assuming that all the energy remaining within the external membrane is lost, one gets an underestimated value of $Q$ around $10^{6}$.

The resonator microfabrication process takes advantage of standard techniques of quartz wet etching, using fluorhydric acid. Starting from a superpolished (down to $2 \AA \mathrm{rms}$ ) ultrapure quartz substrate, the wafer is metallized with a $15-\mathrm{nm}$ thick layer of chromium and a 200-nm layer of gold. These layers are used as a long-time resist mask for the etching of the membrane, down to a thickness as low as $20 \mu \mathrm{m}$. Wet etching ensures quasi perfect

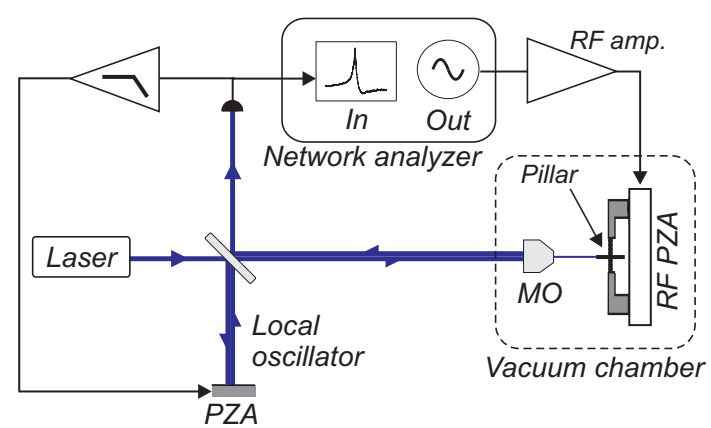

Figure 3. Michelson interferometer setup used to characterize the mechanical properties of the resonator.

symmetry of the pillar with respect to the membrane, which appears as an essential condition to reduce clamping losses and to reach a high $Q$.

Another important condition to reduce mechanical loss is to coat the mirror only on top of the pillar. For that purpose we use a 3D $5-\mu \mathrm{m}$ thick photoresist mask. A photolithography followed by a developer bath allows one to remove the resist mask over a well-defined zone, which is a $60-\mu \mathrm{m}$ diameter spot centered on the top of the pillar. The mirror coated by evaporation technique is a stack of $15 \mathrm{SiO}_{2} / \mathrm{Ta}_{2} \mathrm{O}_{5}$ quarter-wave doublets, with a $100 \mathrm{ppm}$ expected transmission. After coating, the photoresist is removed by standard lift-off techniques, without damaging the optical properties (see Fig. 2d). Measurement of the micropillar reflectivity using a dedicated Fabry-Perot cavity is in progress.

To characterize the mechanical properties of the resonator, a Michelson interferometer with a Nd:YAG laser source has been built (see Fig. 3). The micropillar is mounted on a high-frequency piezoelectric actuator (RF PZA) driven by a high-power RF amplifier. A microscope objective (MO) focuses the laser beam down to a few microns on the sample surface. The whole sensor arm is in a vacuum chamber pumped down to $10^{-3} \mathrm{mBar}$. A photodiode detects the laser intensity at the output of the interferometer, its low-frequency signal being used to control the length of the local oscillator arm through a 


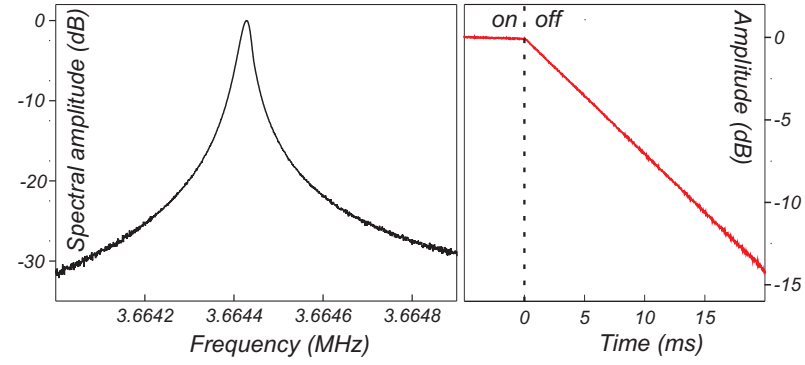

Figure 4. Spectrum of the micropillar mechanical response around its fundamental resonance frequency at $3.66 \mathrm{MHz}$ (left), and ring-down response for the same mechanical mode (right).

feedback loop. The spectral mechanical response of the micropillar is obtained using a network analyzer to synchronously drive the resonator into motion and monitor the radio-frequency photodiode signal.

Fig. 4 (left) presents an example of such a mechanical response spectrum: the mechanical resonance is observed around $3.66 \mathrm{MHz}$, in good agreement with the FEM simulation results. The slight asymmetry of the resonance is attributed to an electrical interference between the optomechanically induced RF signal detected on the photodiode and a spurious modulation radiated by the $\mathrm{RF}$ amplifier. The interferometric measurement being sensitive to longitudinal displacements of the surface, only a few and well-isolated compression-expansion mechanical resonances are observed as expected from FEM simulations, with a dynamical response at least $40 \mathrm{~dB}$ over the mechanical background.

The optical spot can be swept over the whole surface of the resonator, allowing for a mapping of the vibration profile. It is mostly found uniform over the upper surface of the pillar, with a typical displacement amplitude of the order of $5 \mathrm{~nm}$, to be compared to the piezoelectric actuator displacement, measured at a $20 \mathrm{pm}$ level on the substrate.

As the intrinsic interferometer jitter may widen the observed mechanical resonance, its quality factor is measured using a ring-down technique: once the resonator is driven close to its resonance frequency, the actuation is switched off and we record the time evolution of the free resonator motion using a spectrum analyzer in zerospan mode. The observed exponential decay then gives the damping time, hence the quality factor. Results depend on the residual thickness of the membrane: typical values are larger than $10^{5}$, reaching values as large as $Q=1.8 \times 10^{6}$ for the best samples without optical coating. Work is in progress to reduce the membrane thickness and to improve the pillar symmetry, in particular by using optical coatings on both sides.

To conclude, we have developed a new quartz resonator in a compression-expansion mode, suitable for cavity optomechanics. Preliminary mechanical and optical char- acterizations yield promising results for the optomechanical coupling that can be obtained with this device. Next steps will be the operation of the resonator in a dedicated high-finesse Fabry-Perot cavity, inside a dilution fridge working at a base temperature of $30 \mathrm{mK}$. Thanks to the very high mechanical quality factor of the oscillator, we expect to reach a final effective temperature at the $100 \mu \mathrm{K}$ level using laser cooling, thus demonstrating the quantum ground state of such a macroscopic optomechanical system.

The authors acknowledge practical help from J. Teissier for the scanning electron microscope pictures, and financial support of the "Agence Nationale de la Recherche" (ANR) France, Programme blanc, $N^{\circ}$ ANR07-BLAN-2060 ARQOMM and of FP7 Specific Targeted Research Project Minos.

\section{REFERENCES}

${ }^{1}$ X.M.H. Huang, C.A. Zorman, M. Mehregany, and M.L. Roukes, Nature 421, 496 (2003).

${ }^{2}$ M.D. LaHaye, O. Buu, B. Camarota, and K.C. Schwab, Science 304, 74 (2004).

${ }^{3}$ A.D. O'Connell, M. Hofheinz, M. Ansmann, R.C. Bialczak, M. Lenander, E. Lucero, M. Neeley, D. Sank, H. Wang, M. Weides, J. Wenner, J.M. Martinis, and A.N. Cleland, Nature 464, 697 (2010).

${ }^{4}$ J.D. Teufel, T. Donner, D. Li, J.H. Harlow, M.S. Allman, K. Cicak, A.J. Sirois, J.D. Whittaker, K.W. Lehnert, and R.W. Simmonds, arXiv:1103.2144 (2011).

${ }^{5}$ R. Rivière, S. Deléglise, S. Weis, E. Gavartin, O. Arcizet, A. Schliesser, and T.J. Kippenberg, Phys. Rev. A 83, 063835 (2011).

${ }^{6}$ J.D. Teufel, D. Li, M.S. Allman, K. Cicak, A.J. Sirois, J.D. Whittaker, and R.W. Simmonds, Nature 471, 204 (2011).

${ }^{7}$ F. Marquardt and S.M. Girvin, Physics 2, 40 (2009).

${ }^{8}$ M. Pinard, A. Dantan, D. Vitali, O. Arcizet, T. Briant, and A. Heidmann, Europhys. Lett. 72, 747 (2005).

${ }^{9}$ O. Arcizet, P.-F. Cohadon, T. Briant, M. Pinard, and A. Heidmann, Nature 444, 71 (2006).

${ }^{10}$ S. Gigan, H.R. Böhm, M. Paternostro, F. Blaser, G. Langer, J.B. Hertzberg, K.C. Schwab, D. Bäuerle, M. Aspelmeyer, and A. Zeilinger, Nature 444, 67 (2006).

${ }^{11}$ A. Schliesser, O. Arcizet, R. Rivière, G. Anetsberger, and T.J. Kippenberg, Nat. Phys. 5, 509 (2009).

${ }^{12}$ M. Bahriz and O. Le Traon, French Patent FR 1002829, July 2010.

${ }^{13}$ R. Flaminio, J. Franc, C. Michel, N. Morgado, L. Pinard, and B. Sassolas, Class. Quantum Grav. 27, 0840030 (2010).

${ }^{14}$ B. Le Foulgoc, T. Bourouina, O. Le Traon, A. Bosseboeuf, F. Marty, C. Breluzeau, J.-P. Granchamp, and S. Masson, J. Micromech. Microeng. 16, S45 (2006).

${ }^{15}$ J. Lamb and J. Richter, Proc. Roy. Soc. (London) 293A, 479 (1966).

${ }^{16}$ S. Galliou, J. Imbaud, M. Goryachev, R. Bourquin, and P. Abbe, Appl. Phys. Lett. 98, 091911 (2011). 